\title{
Pseudocbetosoma salmonicola Dollfus 1951 et Pseudochetosoma leucisci Ergens 1963 sont-elles des espèces différentes?
}

\author{
Par D. KAKATCHEVA-AVRAMOVA
}

En 1951, Dollfus a décrit l'espèce Ochetosoma salmonicola qu'il plaça en 1952 dans un nouveau genre: Pseudochetosoma Dollfus 1952, de la famille des Steganodermatidae Dollfus 1952 [Trematoda Digenea].

Dollfus a trouvé des Pseudochetosoma salmonicola dans la vésicule biliaire de Poissons d'eau douce : Salmo macrostigma A. Duméril *, Labeobarbus paytoni G.-A. Boulanger et Barbus callensis Cuv. et Val., pris au Maroc.

Plus tard, Ergens a découvert (en 1963) une espèce semblable dans la vésicule biliaire de Leuciscus cephalus albus B., provenant de la rivière Kiri (Albanie septentrionale). En raison de certaines différences dans les dimensions, il l'a prise pour une espèce nouvelle - Pseudochetosoma leucisci Ergens 1963.

En 1964 et 1965, quand nous étions en train d'étudier la faune helminthologique des Poissons des rivières du Balkan occidental, nous avons trouvé pour la première fois dans la vésicule biliaire de certains d'entre eux un Trématode pareil à celui qui a été décrit par Dollfus et Ergens.

Les Poissons dans lesquels nous avons trouvé ce Trématode intéressant par sa localisation sont: Barbus meridionalis petenyi H., Leuciscus cephalus L., Phoxinus phoxinus L., Alburnoides bipunctatus L., Cobitis taenia L., Gobio gobio L., Salmo iridea $\mathrm{F}$. et Nemachilus barbatulus L.

Les rivières où nous avons pêché les poissons porteurs de parasites sont: la Nichava (dans la ville même de Godetch), et son affluent la Vrabnichka (dans la région appelée Mourgachko poïchté), l'Iskretzka (près du village d'Iskretz) et la Barzia (à 3-4 $\mathrm{km}$ de la ville de Berkovitza, près de l'endroit où la Berkovska se jette dans la Barzia).

Nous avons étudié en détail en préparations totales 75 exemplaires à maturité sexuelle, colorés à l'alun-carmin, pris de divers poissons.

* Dollfus a trouvé ce Trématode d'abord dans le gros intestin de Salmo macrostigma A. Duméril mais il estima peu après (1952, p. 371), qu'il y était parvenu en venant de la vésicule biliaire, emplacement normal de l'espèce. 
D'après les recherches que nous avons effectuées et des consultations que nous avons eues avec le Professeur Dollfus (que nous remercions vivement), nous admettons que le Trématode trouvé par nous appartient à l'espèce Pseudochetosoma salmonicola Dollfus 1951.

Les contours des divers organes de nos exemplaires sont pareils et leur disposition est la même chez Pseudochetosoma leucisci et Pseudochetosoma salmonicola (fig. 1, 2 et 3). Dans certains exemplaires, l'œsophage est élargi cylindriquement au milieu, comme chez Pseudochetosoma leucisci. Nous donnons dans le tableau I des dimensions pour comparaison avec celles données respectivement par Dollfus et Ergens.

Les dimensions de nos exemplaires varient sur une plus vaste échelle que celles fournies par Dollfus et Ergens.

La comparaison des indices anatomiques et morphologiques des deux espèces de Pseudochétosomes, décrits par Dollfus et Ergens, montre une grande similitude entre eux, ce qui nous a fait penser qu'il s'agissait de la même espèce.

\section{TABLEAU I}

Comparaison des dimensions (en mm)

de Pseudochetosoma salmonicola (Dollfus 1951),

Pseudochetosoma leucisci (Ergens 1963)

et de Pseudochetosoma salmonicola, trouvé en Bugarie

\begin{tabular}{|c|c|c|c|}
\hline Dimensions & $\begin{array}{l}\text { P. salmonicola } \\
\text { Dollfus } 1951 \\
\text { trouvé au Maroc }\end{array}$ & $\begin{array}{l}P \text {. leucisci } \\
\text { Ergens } 1963 \\
\text { trouvé en } \\
\text { Albanie }\end{array}$ & $\begin{array}{l}\text { P. salmonicola } \\
\text { trouvé en } \\
\text { Bulgarie }\end{array}$ \\
\hline Longueur du corps $\ldots \ldots \ldots$ & $1,370-2,000$ & $2,720-2,850$ & $0,6240-2,500$ \\
\hline Largeur du corps (max.) .... & $0,350-0,410$ & $0,840-1,360$ & $0,3120-1,000$ \\
\hline Longueur du préacetabulum . & $0,130-0,160$ & $0,217-0,258$ & $0,1040-0,2704$ \\
\hline Largeur du préacetabulum ... & $0,130-0,160$ & $0,231-0,299$ & $0,1144-0,2704$ \\
\hline Longueur du pharynx ...... & 0,067 & $0,102-0,122$ & $0,0624-0,1772$ \\
\hline Largeur du pharynx $\ldots \ldots \ldots$. & $0,053-0,060$ & $0,122-0,136$ & $0,0624-0,1456$ \\
\hline Longueur de l'œesophage .... & $0,110-0,210$ & $0,326-0,367$ & $0,1025-0,3120$ \\
\hline Longueur de l'acetabulum ... & $0,220-0,280$ & $0,340-0,380$ & $0,1845-0,4160$ \\
\hline Largeur de l'acetabulum .... & $0,220-0,280$ & $0,312-0,367$ & $0,1558-0,3952$ \\
\hline Longueur de la poche du cirre & $0,150-0,184$ & $0,367-0,421$ & $0,1558-0,3952$ \\
\hline Largeur de la poche du cirre & $0,057-0,068$ & $0,081-0,095$ & $0,0615-0,1040$ \\
\hline Longueur des œufs $\ldots \ldots \ldots$. & $0,075-0,080$ & $0,072-0,081$ & $0,0697-0,0820$ \\
\hline Largeur des œufs ......... & $0,045-0,050$ & $0,033-0,042$ & $0,0246-0,0451$ \\
\hline
\end{tabular}

D'après la diagnose différentielle par Ergens (1963), $P$. leucisci diffère de $P$. salmonicola par les dimensions du corps, du pharynx, du préacetabulum et de l'acetabulum, 


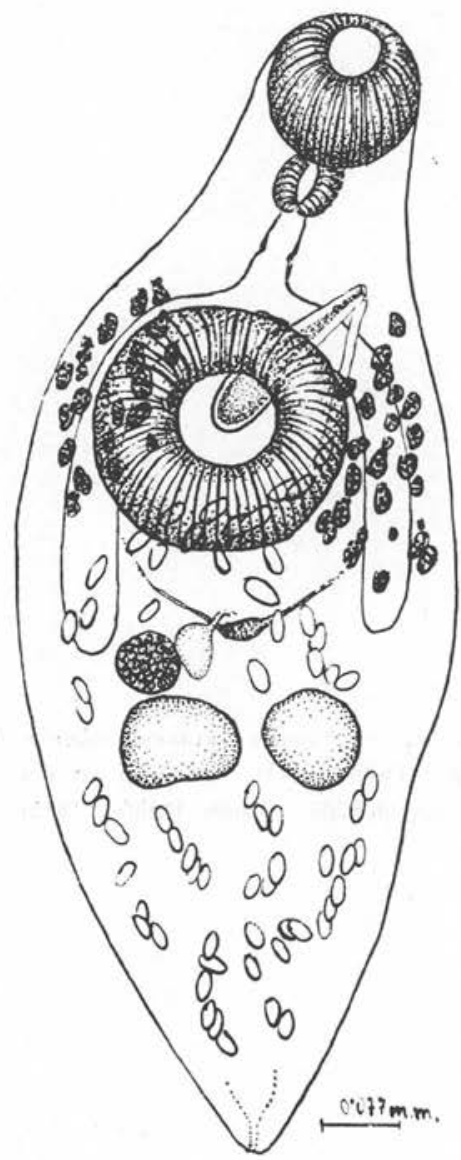

Fig. 1. - Pseudochetosoma salmonicola Dollfus 1951, trouvé en Bulgarie (orig.)

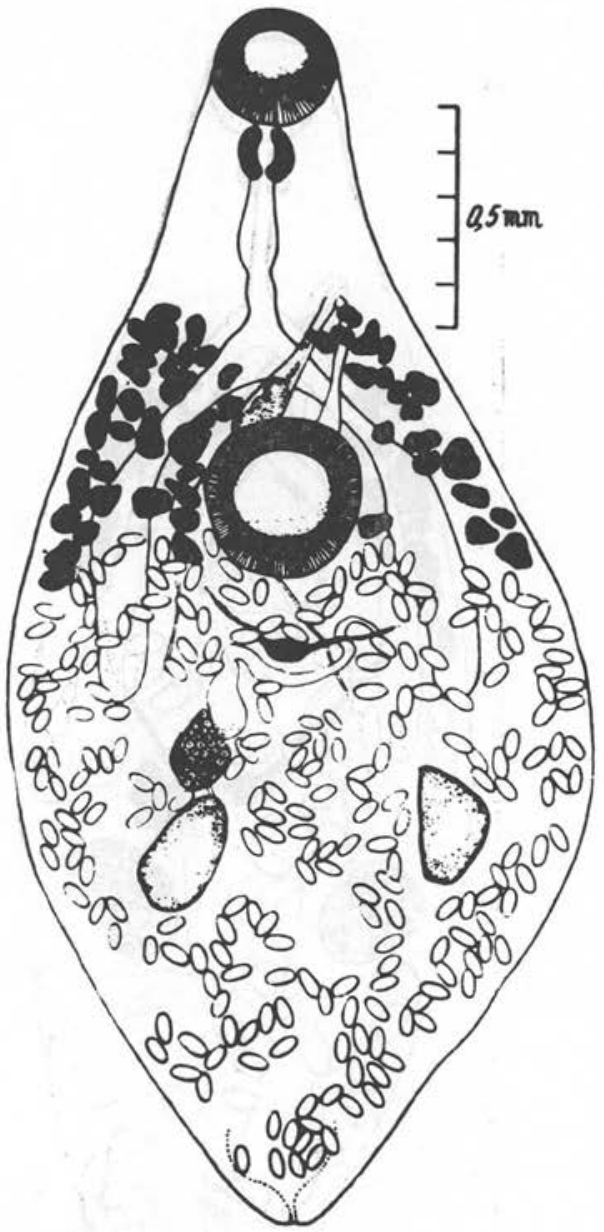

FIG. 2. - Pseudochetosoma leucisci Ergens 1963 (Holotypes, d'après Ergens)

par la longueur de l'œsophage et de la poche de cirre, par le rapport entre la grandeur du préacetabulum et de l'acetabulum et par le contour du corps en forme de bouteille, ainsi que par la forme de l'ovaire et l'élargissement cylindrique de l'œsophage.

Parmi ces différences, les plus essentielles sont : le rapport entre la grandeur du préacetabulum et celle de l'acetabulum, la forme de l'ovaire et l'élargissement cylindrique de l'œsophage.

En ce qui concerne le rapport entre le préacetabulum et l'acetabulum, la différence entre les deux espèces est la suivante : dans l'espèce décrite par Dollfus, ce rapport est 


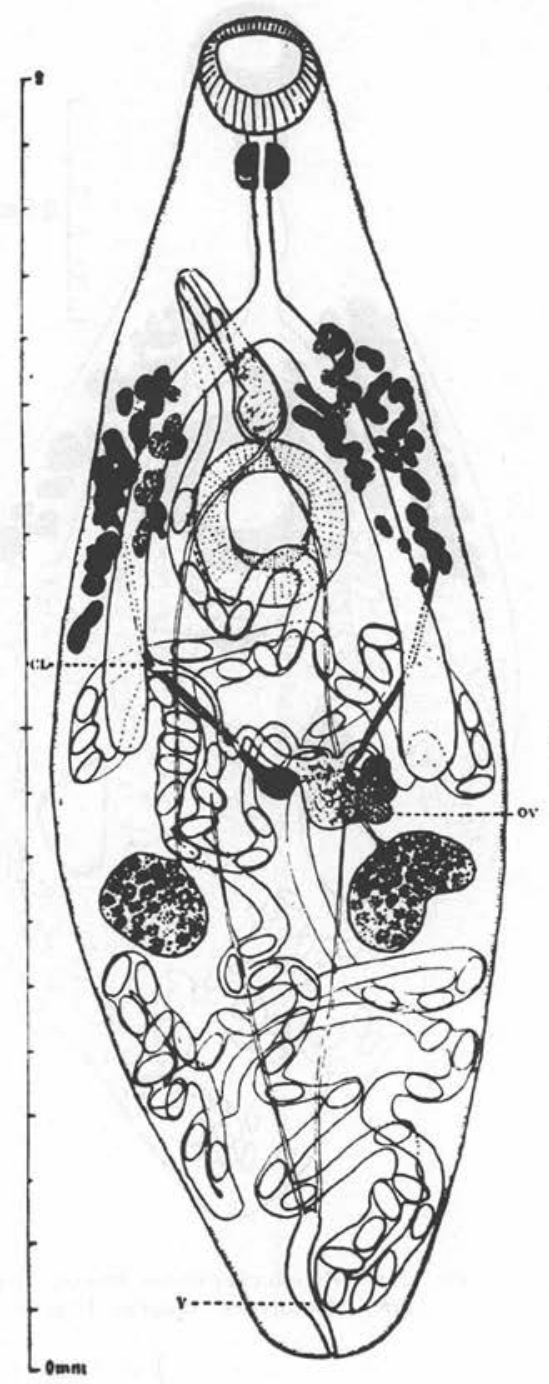

Fig. 3. - Pseudochetosoma salmonicola R.-Ph. Dollfus 1951. Spécimen vu par la face dorsale (d'après Dollfus 1952)

approximativement de 1:2; dans celle décrite par Ergens, il est approximativement de $2: 3$.

Dans notre espèce, il s'est avéré que ce rapport varie. Dans la plupart des cas, il est de $2: 3$, mais il y a aussi des cas où il est de $1: 2$. Il en est ainsi, par exemple, dans les exemplaires parasitant $B$. meridionalis petenyi et $P h$. phoxinus. En ce qui concerne la forme de l'ovaire chez nos exemplaires, il est arrondi, non lobé, comme chez $P$. leucisci. Dollfus observe que dans ses exemplaires l'ovaire est souvent un peu lobé. 
L'élargissement cylindrique de l'œsophage varie également dans certaines limites. Dans notre matériel, cela est plus ou moins nettement exprimé.

Les différences dans les dimensions entre les deux espèces mentionnées dans le tableau $\mathrm{n}^{\circ} \mathrm{I}$ ne sauraient être admises comme un indice sûr de différenciation, car elles n'ont été faites, en ce qui concerne $P$. leucisci, que sur seulement quatre exemplaires. La variation des dimensions dans une échelle plus large dans notre matériel est due, chez la plupart des exemplaires mesurés, à la diversité des hôtes de ce Trématode. Il est intéressant de noter que ce sont les organes des exemplaires à maturité sexuelle trouvés dans Cobitis taenia qui avaient les plus petites dimensions, tandis que ceux des exemplaires trouvés dans Phoxinus phoxinus et $B$. meridionalis petenyi en avaient de plus grandes.

De l'analyse que nous avons faite, nous pouvons conclure que, s'il existe des bases anatomiques et morphologiques pour différencier les formes de Pseudochétosomes, l'espèce décrite par Ergens doit néanmoins être rapportée à Ps. salmonicola. Il est nécessaire toutefois d'étendre les caractéristiques de l'espèce, car ses dimensions varient dans des limites plus larges que celles observées jusqu'à présent.

Diagnose supplémentée de Ps. salmonicola R. Ph. Dollfus.

Distome euryadaptif en ce qui concerne son hôte définitif. Il est parasite dans la vésicule biliaire de Labeobarbus paytoni, Barbus callensis, Salmo macrostigma, dans l'espèce Leuciscus cephalus, également dans la forme $L$. cephalus albus, dans B. meridionalis petenyi, Alb. bipunctatus, Gobio gobio, Ph. phoxinus, Salmo irideus, Cobitis taenia et Nemachilus barbatulus. Il atteint une longueur de 0,624 à $2,85 \mathrm{~mm}$. Sa largeur maximum atteint de 0,312 à $1,36 \mathrm{~mm}$. La longueur de son préacetabulum varie de 0,1040 à $0,2704 \mathrm{~mm}$, et sa largeur de 0,1144 à $0,2704 \mathrm{~mm}$. Son acetabulum a de 0,1845 à $0,4160 \mathrm{~mm}$ de long et de 0,1558 à $0,3952 \mathrm{~mm}$ de large. La longueur du pharynx est de 0,0624 à $0,1772 \mathrm{~mm}$, la largeur du pharynx de 0,0530 à $0,1457 \mathrm{~mm}$. La longueur de l'œsophage de 0,1025 à $0,3952 \mathrm{~mm}$; la longueur de la poche de cirre de 0,1558 à $0,3952 \mathrm{~mm}$, et sa largeur de 0,057 à $0,104 \mathrm{~mm}$; la longueur des œufs de 0,0697 à $0,0820 \mathrm{~mm}$, et leur largeur de 0,0246 à $0,0500 \mathrm{~mm}$. Les contours de l'ovaire sont ou bien entiers ou bien un peu lobés.

\section{Bibliographie}

1. Dollfus (Robert-Ph.), 1952. - Affinités naturelles de Pseudochetosoma salmonicola R. $\mathrm{Ph}$. Dollfus 1951 (Famille Stegonodermatidea nov.). Emendation de la superfamille Haploporoidea. W. Nicoll 1935. Archives de l'Institut Pasteur du Maroc, tome IV, cahier 5, p. 369-386, 1 fig.

2. Ergens (R.), 1963. - Ueber Pseudochetosoma leucisci n. sp. (Trematoidea) und Ancyrocephalus cobitis n. sp. (Monogenoidea), zwei neue parasitische Würmer der Fische Albaniens. Zeitschrift für Parasitenkunde, 22 Band, 4 Heft, 287-291.

[Laboratoire central d'Helminthologie de l'Académie bulgare des Sciences, Sofia (Bulgarie) (Professeur K. Matoff)] 